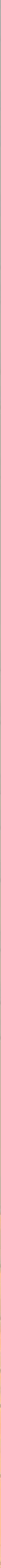




\title{
Annihilating the cohomology of group schemes
}

\author{
Bhargav Bhatt
}

\begin{abstract}
Our goal in this note is to show that cohomology classes with coefficients in finite flat group schemes can be killed by finite covers of the base scheme, and similarly for abelian schemes with "finite covers" replaced by "proper covers." We apply this result to commutative algebra to give by a conceptual proof of Hochster-Huneke's theorem on the existence of big Cohen-Macaulay algebras in positive characteristic; all previous proofs of this result were equational or cocycle-theoretic in nature.
\end{abstract}

\section{Introduction}

Given a scheme $S$ with a sheaf $G$ and class $\alpha \in H^{n}(S, G)$ for $n>0$, a natural question one may ask is if there exist covers $\pi: T \rightarrow S$ such that $\pi^{*} \alpha=0$ ? Of course, as stated, the answer is trivially yes as we may take $T$ to be a disjoint union of suitable opens occurring in a Čech cocycle representing $\alpha$. However, the question becomes interesting if we require geometric conditions on $\pi$, such as properness or even finiteness. Our goal is to study such questions for fppf cohomology in the case that $G$ is either a finite flat commutative group scheme or an abelian scheme. Our main results are:

Theorem 1.1. Let $S$ be a noetherian excellent scheme, and let $G$ be a finite flat commutative group scheme over $S$. Then classes in $H_{\mathrm{fppf}}^{n}(S, G)$ can be killed by finite surjective maps to $S$ for $n>0$.

Theorem 1.2. Let $S$ be a noetherian excellent scheme, and let $A$ be an abelian scheme over $S$. Then classes in $H_{\mathrm{fppf}}^{n}(S, A)$ can be killed by proper surjective maps to $S$ for $n>0$. Moreover, there exists an example of a normal affine scheme $S$ that is essentially of finite type over $\mathbb{C}$, and an abelian scheme $A \rightarrow S$ with a class in $H_{\mathrm{fppf}}^{1}(S, A)$ that cannot be killed by finite surjective maps to $S$.

MSC2010: primary 14L15; secondary 13D45, 14K05, 14F20.

Keywords: group schemes, abelian varieties, étale cohomology, fppf cohomology, big

Cohen-Macaulay algebras. 
We stress that there are no assumptions on the residue characteristics of $S$ in either theorem above.

Our primary motivation for proving the preceding results was to obtain a better understanding of the Hochster-Huneke proof of the existence of big CohenMacaulay algebras in positive characteristic commutative algebra; see [Hochster and Huneke 1992]. We have succeeded in this endeavour as we can give a new and essentially topological proof of the Hochster-Huneke result by using the cohomology-annihilation results discussed above in lieu of the more traditional equational approaches; see Section 5 for more. We are hopeful that a similar approach, coupled with the constructions in [Fontaine 1994] of mixed characteristic rings admitting Frobenius actions, will eventually provide an approach to Hochster's homological conjectures in mixed characteristic commutative algebra; we refer the interested reader to [Hochster 2007] for further information.

An informal summary of the proofs: To prove Theorem 1.1, we first use a theorem of Raynaud to embed a finite flat group scheme into an abelian scheme; this permits a reduction to from fppf cohomology to étale cohomology by a theorem of Grothendieck. Next, using an observation due to Gabber, we reduce from étale cohomology to Zariski cohomology, and then we solve the problem by hand. For Theorem 1.2, we reduce as before to Zariski cohomology, and then solve the problem using de Jong's alterations results combined with an observation concerning rational sections of an abelian scheme over a regular base scheme. The example referred to in Theorem 1.2 is discussed in Section 6, and relies on a construction of Raynaud. Lastly, the Hochster-Huneke theorem is reproved by first reformulating it as a suitable cohomology-annihilation statement for the higher local cohomology of the structure sheaf, and then deducing this statement from Theorem 1.1 by using finite flat subgroup schemes of $\mathbb{G}_{a}$ defined by additive polynomials in Frobenius.

Notations and conventions. All group schemes occurring in this note are commutative; all the cohomology groups occurring in this note are computed in the fppf topology unless otherwise specified. For a scheme $X$, the big site of $X$ equipped with the étale topology is denoted $(\mathrm{Sch} / X)_{\text {ét }}$, while the small site is denoted $X_{\text {ét }}$; similarly for other topologies like the fppf and Zariski topologies.

Organisation of this note. In Section 2 we recall Gabber's observation alluded to above. Using this observation, we prove Theorem 1.1 in Section 3, and the first half of Theorem 1.2 in Section 4. Next, in Section 5, we explain how to use Theorem 1.1 to give a new proof of the Hochster-Huneke theorem. We close in Section 6 by giving an example that illustrates the necessity of "proper" in the first half of Theorem 1.2 and finishes its proof. 


\section{An observation of Gabber}

In this section, we recall a result of Gabber concerning the local structure of the étale topology. This observation permits reduction of étale cohomological considerations to those in finite flat cohomology and those in Zariski cohomology. We begin with an elementary lemma on extending covers that will be used repeatedly in the sequel.

Lemma 2.1. Fix a noetherian scheme $X$. Given an open dense subscheme $U \rightarrow X$ and a finite (surjective) morphism $f: V \rightarrow U$, there exists a finite (surjective) morphism $\bar{f}: \bar{V} \rightarrow X$ such that $\bar{f}_{U}$ is isomorphic to $f$. Given a Zariski open cover $U=\left\{j_{i}: U_{i} \rightarrow X\right\}$ with a finite index set, and finite (surjective) morphisms $f_{i}: V_{i} \rightarrow U_{i}$, there exists a finite (surjective) morphism $f: Z \rightarrow X$ such that $f_{U_{i}}$ factors through $f_{i}$. The same claims hold if "finite (surjective)" is replaced by "proper (surjective)" everywhere.

Proof. We first explain how to deal with the claims for finite morphisms. For the first part, Zariski's main theorem [Grothendieck 1966, Théorème 8.12.6] applied to the morphism $V \rightarrow X$ gives a factorisation $V \hookrightarrow W \rightarrow X$ where $V \hookrightarrow W$ is an open immersion, and $W \rightarrow X$ is a finite morphism. The scheme-theoretic closure $\bar{V}$ of $V$ in $W$ provides the required compactification in view of the fact that finite morphisms are closed.

For the second part, by the above, we may extend each $j_{i} \circ f_{i}: V_{i} \rightarrow X$ to a finite surjective morphism $\bar{f}_{i}: \bar{V}_{i} \rightarrow X$ such that $\bar{f}_{i}$ restricts to $f_{i}$ over $U_{i} \hookrightarrow X$. Setting $W$ to be the fibre product over $X$ of all the $\bar{V}_{i}$ is then seen to solve the problem.

To deal with the case of proper (surjective) morphisms instead of finite (surjective), we repeat the same argument as above replacing the reference to Zariski's main theorem by one to Nagata's compactification theorem; see [Conrad 2007, Theorem 4.1].

Next, we state Gabber's result (see [Hoobler 1982, Lemma 5; Stacks, 02LH]):

Lemma 2.2. Let $f: U \rightarrow X$ be a surjective étale morphism of affine schemes. Then there exists a finite flat map $g: X^{\prime} \rightarrow X$, and a finite Zariski open cover $\left\{U_{i} \hookrightarrow X^{\prime}\right\}$ such that the natural map $\bigsqcup_{i} U_{i} \rightarrow X$ factors through $U \rightarrow X$.

For completeness, we sketch a proof when $X$ is local; this will be enough for applications.

Sketch of proof. We only explain the proof when $X=\operatorname{Spec}(A)$ is the spectrum of a local ring $A$, and $U=\operatorname{Spec}(B)$ is the spectrum of a local étale $A$-algebra $B$. The structure theorem for étale morphisms (see [Grothendieck 1962, Exposé I, Théorème 7.6]) implies that $B=C_{\mathfrak{m}}$ where

$$
C=A[x] /(f(x)) \text { with } f(x)=x^{n}+a_{1} x^{n-1}+\cdots+a_{n}
$$


a monic polynomial, and $\mathfrak{m} \subset C$ a maximal ideal with $f^{\prime}(x) \notin \mathfrak{m}$. We define

$$
D=A\left[x_{1}, \ldots, x_{n}\right] /\left(\sigma_{i}\left(x_{1}, \ldots, x_{n}\right)-(-1)^{n-i} a_{i}\right)
$$

where $\sigma_{1}, \ldots, \sigma_{n}$ are the elementary symmetric polynomials in the $x_{i}$. This ring is finite free over $A$ of rank $n$ !, admits an action of $S_{n}$ that is transitive on the maximal ideals, and formalises the idea that the coefficients of $f(x)$ can be written as elementary symmetric functions in its roots. In particular, there is a natural morphism $C \rightarrow D$ sending $x$ to $x_{1}$. As both $C$ and $D$ are finite free over $A$, there is a maximal ideal $\mathfrak{m}_{1} \subset D$ lying over $\mathfrak{m} \subset C$. Thus, there is a natural map $a: B \rightarrow D_{\mathfrak{m}_{1}}$. By the $S_{n}$-action, for every maximal ideal $\mathfrak{n} \subset D$, there is an automorphism $D \rightarrow D$ sending $\mathfrak{m}_{1}$ to $\mathfrak{n}$. Composing such an automorphism with $a$, we see that for every maximal ideal $\mathfrak{n} \subset D$, the structure map $A \rightarrow D_{\mathfrak{n}}$ factorises through $A \rightarrow B$ for some map $B \rightarrow D_{\mathfrak{n}}$; the claim follows.

Actually, we use a slight weakening of Gabber's result - relaxing finite flat to finite surjective - that remains true when the schemes under consideration are no longer assumed to be affine.

Lemma 2.3. Let $f: U \rightarrow X$ be a surjective étale morphism of noetherian schemes. Then there exists a finite surjective map $g: X^{\prime} \rightarrow X$, and a finite Zariski open cover $\left\{U_{i} \hookrightarrow X^{\prime}\right\}$ such that the natural map $\bigsqcup_{i} U_{i} \rightarrow X$ factors through $U \rightarrow X$.

Proof. We can solve the problem locally on $X$ by Lemma 2.2 and a "smearing out" argument. This means that there exists a Zariski open cover $\left\{V_{i} \hookrightarrow X\right\}$, finite surjective (even flat) maps $W_{i} \rightarrow V_{i}$, and Zariski covers $\left\{Y_{i j} \hookrightarrow W_{i}\right\}$ such that $\sqcup Y_{i j} \rightarrow V_{i}$ factors through $U \times_{X} V_{i} \rightarrow V_{i}$. By Lemma 2.1, we may find a single finite surjective map $W \rightarrow X$ such that $W \times{ }_{X} V_{i} \rightarrow V_{i}$ factors through $W_{i} \rightarrow V_{i}$. Setting $X^{\prime}=W$ and pulling back the covers $\left\{Y_{i j} \rightarrow W_{i}\right\}$ to $W \times{ }_{X} V_{i}$ then solves the problem.

\section{The theorem for finite flat commutative group schemes}

In this section we prove Theorem 1.1 following the plan explained in the introduction. To carry that program out, we first explain how to relate the fppf cohomology of finite flat group schemes to étale cohomology; it turns out that they are almost the same.

Proposition 3.1. Let $S$ be the spectrum of a strictly henselian local ring, and let $G$ be a finite flat commutative group scheme over $S$. Then $H^{i}(S, G)=0$ for $i>1$.

Proof. We first explain the idea informally. Using a theorem of Raynaud, we can embed $G$ into an abelian scheme, which allows us to express the cohomology of $G$ in terms of that of abelian schemes. As abelian schemes are smooth, a result of Grothendieck ensures that their fppf cohomology coincides with their étale 
cohomology. As the latter vanishes when $S$ is strictly henselian, we obtain the desired conclusion.

Now for the details: a construction of Raynaud (see [Berthelot et al. 1982, Théorème 3.1.1]) gives the existence of an abelian scheme $A \rightarrow S$ and an $S$-closed immersion $G \hookrightarrow A$ of group schemes. By Deligne's theorem [Tate and Oort 1970, $\S 1$, Theorem], we have $G \subset A[n]$ where $n$ is the order of $G$. The quotient map $A / G \rightarrow A / A[n] \simeq A$ of fppf sheaves is an $A[n] / G$-torsor. Since $A[n] / G$ is a finite group scheme [Raynaud 1967, Théorème $1.1(\mathrm{v})$ ], the map $A / G \rightarrow A$ is fppf locally representable by a finite morphism of schemes. Since the quotient $A$ is a scheme, fppf descent for finite morphisms shows that $A / G$ is also a scheme. The map $A / G \rightarrow A$ is finite, so $A / G$ is proper over $S$ and acquires the structure of an $S$-group scheme by functoriality. Using the faithful flatness of $A \rightarrow A / G$ (as it is a $G$-torsor) and $A \rightarrow S$, one concludes:

- $A / G \rightarrow S$ is faithfully flat by an elementary flatness argument.

- $A / G \rightarrow S$ has geometrically regular fibres as these fibres admit a finite flat cover that is smooth.

- $A / G \rightarrow S$ has geometrically connected fibres as these fibres are dominated by those of $A \rightarrow S$.

These properties show that $A / G \rightarrow S$ is an abelian scheme. Hence, we have a short exact sequence

$$
0 \rightarrow G \rightarrow A \rightarrow A / G \rightarrow 0
$$

of abelian sheaves on the fppf site of $S$ relating the finite flat commutative group scheme $G$ to the abelian schemes $A$ and $A / G$. This gives rise to a long exact sequence

$$
\cdots \rightarrow H^{n-1}(S, A / G) \rightarrow H^{n}(S, G) \rightarrow H^{n}(S, A) \rightarrow \cdots
$$

of fppf cohomology groups. By Grothendieck's theorem [1968b, Théorème 11.7], fppf cohomology coincides with étale cohomology when the coefficients are smooth group schemes. Applying this to $A$ and $A / G$ shows $H^{i}(S, A)=H^{i}(S, A / G)=0$ for $i>0$ as $S$ is strictly henselian. The claim about $G$ now follows from the preceding exact sequence.

Remark 3.2. Proposition 3.1 may be reformulated topologically to say for a scheme $X$ and a finite flat group scheme $G \rightarrow X$, we have $\mathrm{R}^{i} f_{*} G=0$ for $i \geq 2$, where $f:(\mathrm{Sch} / X)_{\mathrm{fppf}} \rightarrow(\mathrm{Sch} / X)_{\text {ét }}$ is the morphism of (big) topoi defined by viewing étale covers as fppf covers. The Leray spectral sequence then reduces to a long exact sequence

$$
\cdots \rightarrow H_{\mathrm{et}}^{i}(X, G) \rightarrow H_{\mathrm{fppf}}^{i}(X, G) \rightarrow H_{\mathrm{et}}^{i-1}\left(X, \mathrm{R}^{1} f_{*} G\right) \rightarrow \cdots .
$$


Next, we explain how to deal with Zariski cohomology with coefficients in a finite flat group scheme.

Proposition 3.3. Let $S$ be a normal noetherian scheme, and let $G \rightarrow S$ be a finite flat commutative group scheme. Then $H_{\mathrm{Zar}}^{n}(S, G)=0$ for $n>0$.

Proof. We may assume that $S$ is connected. As constant sheaves on irreducible topological spaces are acyclic, it will suffice to show that $G$ restricts to a constant sheaf on the small Zariski site of $S$, that is, that the restriction maps $G(S) \rightarrow G(U)$ are bijective for any nonempty open subset $U \hookrightarrow S$. Injectivity follows from the density of $U \hookrightarrow S$ and the separatedness of $G \rightarrow S$. To show surjectivity, we note that given a section $U \rightarrow G$ of $G$ over $U$, we can simply take the scheme-theoretic closure of $U$ in $G$ to obtain an integral closed subscheme $S^{\prime} \hookrightarrow G$ such that the projection map $S^{\prime} \rightarrow S$ is finite and an isomorphism over $U$. By the normality of $S$, this forces $S^{\prime}=S$. Thus, $G$ restricts to a constant sheaf on $S$, as claimed.

We can now complete the proof of Theorem 1.1 by following the outline sketched in the introduction.

Proof of Theorem 1.1. Let $S$ be a noetherian excellent scheme, and let $G \rightarrow S$ be a finite flat commutative group scheme. We need to show that classes in $H^{n}(S, G)$ can be killed by finite covers for $S$ for $n>0$. We deal with the $n=1$ case on its own, and then proceed inductively.

For $n=1$, note that classes in $H^{1}(S, G)$ are represented by fppf $G$-torsors $T$ over $S$. By faithfully flat descent for finite flat morphisms, such schemes $T \rightarrow S$ are also finite flat. Passing to the total space of $T$ trivialises the $G$-torsor $T$. Therefore, classes in $H^{1}(S, G)$ can be killed by finite flat covers of $S$.

We now fix an integer $n>1$ and a cohomology class $\alpha \in H^{n}(S, G)$. By Proposition 3.1, we know that there exists an étale cover of $S$ over which $\alpha$ trivialises. By Lemma 2.3, after replacing $S$ by a finite cover, we may assume that there exists a Zariski cover $\mathcal{U}=\left\{U_{i} \hookrightarrow S\right\}$ such that $\left.\alpha\right|_{U_{i}}$ is Zariski locally trivial. The Čech spectral sequence for this cover is

$$
H^{p}\left(U, H^{q}(G)\right) \Rightarrow H^{p+q}(S, G)
$$

where $H^{q}(G)$ is the Zariski presheaf $V \mapsto H^{q}(V, G)$. By construction, the class $\alpha$ comes from some $\alpha^{\prime} \in H^{n-q}\left(\mathcal{U}, H^{q}(G)\right)$ with $q<n$. The group $H^{n-q}\left(u, H^{q}(G)\right)$ is the $(n-q)$-th cohomology group of the standard Čech complex

$$
\prod_{i} H^{q}\left(U_{i}, G\right) \rightarrow \prod_{i<j} H^{q}\left(U_{i j}, G\right) \rightarrow \cdots
$$

By the inductive assumption and the fact that $q<n$, terms of this complex can be annihilated by finite covers of the corresponding schemes. By Lemma 2.1, we may refine these finite covers by one that comes from all of $S$. In other words, we can find 
a finite surjective cover $S^{\prime} \rightarrow S$ such that $\left.\alpha^{\prime}\right|_{S^{\prime}}=0$. After replacing $S$ with $S^{\prime}$, the Čech spectral sequence then implies that $\alpha$ comes from some $H^{n-q^{\prime}}\left(U, H^{q^{\prime}}(G)\right)$ with $q^{\prime}<q$. Proceeding in this manner, we can reduce the second index $q$ all the way down to 0 , that is, assume that the class $\alpha$ lies in the image of the map

$$
H^{n}(U, G) \rightarrow H^{n}(S, G) .
$$

Now we are reduced to the situation in Zariski cohomology that was tackled in Proposition 3.3.

Remark 3.4. The proof given above for Theorem 1.1 used the intermediary of abelian schemes to connect fppf cohomology and étale cohomology with coefficients in a finite flat commutative group scheme $G$ (see Proposition 3.1). When the coefficient group scheme $G$ is smooth (or equivalently étale), this reduction follows directly from Grothendieck's theorem. In general, one can avoid abelian schemes by using a trick due to Messing to embed the group scheme in a smooth affine group: any commutative finite flat $S$-group scheme $G$ may be realised as a closed subgroup of $A=\operatorname{Res}_{G^{\vee} / S}\left(\mathbb{G}_{m}\right)$ where $G^{\vee}$ denotes the Cartier dual of $G$; the map $G \rightarrow A$ is the tautological one coming from the definition $G^{\vee}=\operatorname{Hom}\left(G, \mathbb{G}_{m}\right)$; see [Messing 1972, §IV.1] for more. One can then show that $A$ and $A / G$ are $S$-smooth and representable, so the rest of the proof of Proposition 3.1 goes through. We thank Brian Conrad for pointing this out.

Remark 3.5. If $G$ is a finite flat group scheme over $S$ which is not necessarily abelian, the $H^{1}$ part of Theorem 1.1 remains valid since one can trivialise a $G$-torsor $\pi: T \rightarrow S$ using the finite flat morphism $\pi$.

Example 3.6. We give an example showing that Zariski, étale, and fppf cohomologies can differ. Let $k=\mathbb{F}_{p}$, and $G=\mu_{p} \times \mu_{n}$ where $n$ is prime to $p$.

- $H_{\text {Zar }}^{1}(\operatorname{Spec}(k), G)=0$. Indeed, $\operatorname{Spec}(k)$ is a Zariski point, so the higher (Zariski) cohomology of all sheaves vanishes.

- $H_{\text {ét }}^{1}(\operatorname{Spec}(k), G)=k^{*} /\left(k^{*}\right)^{n}$. This follows from the Kummer sequence

$$
0 \rightarrow \mu_{n} \rightarrow \mathbb{G}_{m} \rightarrow \mathbb{G}_{m} \rightarrow 0,
$$

Hilbert's theorem 90, and the fact that $\mu_{p} \simeq 0$ on the small étale site of $k$.

- $H_{\mathrm{fppf}}^{1}(\operatorname{Spec}(k), G)=k^{*} /\left(k^{*}\right)^{n} \times k^{*} /\left(k^{*}\right)^{p}$. This follows from the Kummer sequence for both $\mu_{n}$ and $\mu_{p}$; we need the flat topology to get right exactness of the Kummer sequence for $\mu_{p}$.

\section{The theorem for abelian schemes}

Our goal in this section is to prove the first half of Theorem 1.2. The arguments here essentially mirror those for finite flat commutative group schemes presented in 
Section 3. The key difference is that annihilating Zariski cohomology requires more complicated constructions when the coefficients are abelian schemes. We handle this by proving a generalisation of Weil's extension lemma (see Proposition 4.2). This generalisation requires strong regularity assumptions on $S$ and is one of the two places in our proof of Theorem 1.2 that we need proper covers instead of finite ones; the other is the case of $H^{1}$.

We begin by recording an elementary criterion for a map to an abelian variety to be constant.

Lemma 4.1. Let $A$ be an abelian variety over an algebraically closed field $k$, and let $C$ be a reduced variety over $k$. Fix an integer $\ell$ invertible on $k$. A map $g: C \rightarrow A$ is constant if and only if it induces the 0 map $H_{e t}^{1}\left(A, \mathbb{Q}_{\ell}\right) \rightarrow H_{e ̂ t}^{1}\left(C, \mathbb{Q}_{\ell}\right)$.

Proof. It suffices to show that a map like $g$ that induces the 0 map on $H^{1}$ is trivial. As any $k$-variety is covered by curves, it suffices to show that the map $g$ is constant on all curves in $C$. Thus, we reduce to the case that $C$ is a curve. We may also clearly assume that $C$ is normal, that is, smooth. Let $\bar{C}$ denote the canonical smooth projective model of $C$. Since $A$ is proper, the map $g$ factors through a map $\bar{g}: \bar{C} \rightarrow A$. Since $C$ and $\bar{C}$ are normal, the map $\pi_{1}(C) \rightarrow \pi_{1}(\bar{C})$ is surjective. Hence, the map $H_{\text {ét }}^{1}\left(\bar{C}, \mathbb{Q}_{\ell}\right) \rightarrow H_{\text {ét }}^{1}\left(C, \mathbb{Q}_{\ell}\right)$ is injective. Thus, to answer the question, we may assume that $C=\bar{C}$ is a smooth projective curve.

Let $A \hookrightarrow \mathbb{P}^{n}$ be a closed immersion corresponding to a very ample line bundle $\mathscr{L}$. The map $g: C \rightarrow A$ will be constant if we can show that $g^{*} \mathscr{L}$ is not ample, that is, has degree 0 . As the $\ell$-adic cohomology of an abelian variety is generated in degree 1 (see [Milne 2008, §12]), the hypothesis on $H^{1}$ implies that the map $H_{\text {ett }}^{2}\left(A, \mathbb{Q}_{\ell}\right) \rightarrow H^{2}\left(C, \mathbb{Q}_{\ell}\right)$ is also 0. In particular, $g^{*}\left(c_{1} \mathscr{L}\right)=0$, where $c_{1}(\mathscr{L}) \in$ $H^{2}\left(A, \mathbb{Q}_{\ell}(1)\right) \simeq H^{2}\left(A, \mathbb{Q}_{\ell}\right)$ is the first Chern class of the line bundle $\mathscr{L}$. Since applying $g^{*}$ commutes with taking the first Chern class, it follows that $c_{1}\left(g^{*} \mathscr{L}\right)=0$, hence $g^{*} \mathscr{L}$ has degree 0 as desired.

We now prove the promised extension theorem for maps into abelian schemes.

Proposition 4.2. Let $S$ be a regular connected excellent noetherian scheme, and let $f: A \rightarrow S$ be an abelian scheme. For any nonempty open $U \subset S$, the restriction map $A(S) \rightarrow A(U)$ is bijective.

Proof. Let $j: U \rightarrow S$ denote the open immersion defined by $U$. The bijectivity of $A(S) \rightarrow A(U)$ will follow by taking global sections if we can show that the natural map of presheaves $a: A \rightarrow j_{*}\left(\left.A\right|_{U}\right)$ is an isomorphism on the small Zariski site of $S$. As both the source and the target of $a$ are actually sheaves for the étale topology on $S$, we may localise to assume that $S$ is the spectrum of a strictly henselian local ring $R$. In this setting, we will show that $A(S) \rightarrow A(U)$ is bijective using $\ell$-adic cohomology. 
The injectivity of $A(S) \rightarrow A(U)$ follows from the density of $U \subset S$ and the separatedness of $A \rightarrow S$. To show surjectivity, by the valuative criterion of properness, we may assume that the complement $S \backslash U$ has codimension at least 2 in $S$. Let $s: U \rightarrow A$ be a section of $A$ over $U$. By taking the normalised scheme-theoretic closure of $s(U) \subset A$, we obtain a proper birational map $p: S^{\prime} \rightarrow S$ that is an isomorphism over $U$, and an $S$-map $i: S^{\prime} \rightarrow A$ extending $s$ over $U$. The desired surjectivity then reduces to showing that $i$ is constant on the fibres of $p$. Since $p_{*} \mathrm{O}_{S^{\prime}}=\mathrm{O}_{S}$, the rigidity lemma (see [Mumford et al. 1994, Proposition 6.1]) shows that it suffices to show that $i$ collapses the reduced special fibre $S_{s}^{\prime}$, where $s \in S$ is the closed point. By Lemma 4.1, it is enough to check that the induced map $H^{1}\left(A_{s}, \mathbb{Q}_{\ell}\right) \rightarrow H^{1}\left(S_{s}^{\prime}, \mathbb{Q}_{\ell}\right)$ is trivial for some integer $\ell$ invertible on $S$. Note that we have the following commutative diagram:

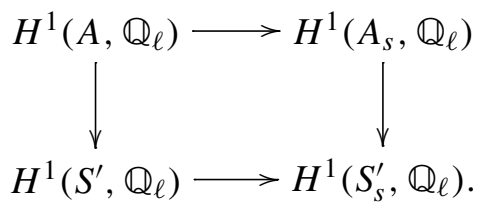

The horizontal maps are isomorphisms by the proper base change theorem in étale cohomology (see [Deligne 1977, Arcata IV-1, Théorème 1.2]) as $S$ is a strictly henselian local scheme. Hence, it suffices to show that $H^{1}\left(A, \mathbb{Q}_{\ell}\right) \rightarrow H^{1}\left(S^{\prime}, \mathbb{Q}_{\ell}\right)$ is 0 . Since $H^{1}\left(S^{\prime}, \mathbb{Q}_{\ell}\right)=\operatorname{Hom}_{\text {conts }}\left(\pi_{1}\left(S^{\prime}\right), \mathbb{Q}_{\ell}\right)$, it suffices to check that $\pi_{1}\left(S^{\prime}\right)=0$. As $S^{\prime}$ is normal, we know that $\pi_{1}(U) \rightarrow \pi_{1}\left(S^{\prime}\right)$ is surjective. Moreover, by ZariskiNagata purity (see [Grothendieck 1968a, Exposé X, Théorème 3.4]), we know that $\pi_{1}(U) \simeq \pi_{1}(S)$ since $S \backslash U$ has codimension $\geq 2$ in $S$. Since $S$ is strictly henselian, we have $\pi_{1}(S)=0$ and hence $\pi_{1}\left(S^{\prime}\right)=0$ as desired.

Remark 4.3. The main idea for the proof of Proposition 4.2 comes from obstruction theory in topology. Consider the universal family $\pi: U_{g} \rightarrow \mathscr{A}_{g}$ of abelian varieties over the stack $\mathscr{A}_{g}$ of abelian varieties. Proposition 4.2 can be rephrased as asking if every map $S \rightarrow \mathscr{A}_{g}$ with a specified lift $U \rightarrow \mathcal{U}_{g}$ over a dense open $U \subset S$ admits an extension $S \rightarrow \mathcal{U}_{g}$ provided $S$ is smooth. Since the stack $\mho_{g}$ is a classifying space for its fundamental group (since the same is true for $\mathscr{A}_{g}$ and the fibres of $\pi$ ), the answer at the level of homotopy types would be yes if and only if $\pi_{1}(U) \rightarrow \pi_{1}\left(u_{g}\right)$ factors through $\pi_{1}(U) \rightarrow \pi_{1}(S)$. This is essentially what is verified above using purity; Lemma 4.1 allows us to go from this homotopy-theoretic conclusion to a geometric one.

Remark 4.4. Proposition 4.2 can be considered a generalisation of Weil's extension lemma when applied to abelian varieties. Recall that this lemma says that the domain of definition of rational maps from a smooth variety to a group variety has pure codimension 1 . In case the target is proper, that is, an abelian variety $A$, this reduces 
to the statement that $A(X) \simeq A(U)$ for any smooth variety $X$, and dense open $U \hookrightarrow X$.

Remark 4.5. Our proof of Proposition 4.2 is topological as explained in Remark 4.3. As pointed out to us by János Kollár after the present work was completed, one can also give a more geometric proof of Proposition 4.2 as follows: a theorem of Abhyankar (see [Kollár 1996, §VI.1, Theorem 1.2]) implies that for any proper modification $p: S^{\prime} \rightarrow S$ with $S$ noetherian regular excellent, the positive dimensional fibres of $p$ contain nonconstant rational curves. Applying this theorem to the closure $S^{\prime}$ of the graph of a rational map defined by a section $U \rightarrow A$ over an open $U \subset S$ gives our desired claim as abelian varieties do not contain rational curves. We prefer the cohomological approach as a slight variation on it (using cohomology of the structure sheaf $\mathbb{O}_{S}$ instead of the constant sheaf in the proof of Proposition 4.2 and Lemma 4.1) shows that Proposition 4.2 remains valid in characteristic 0 if $S$ has rational singularities. This also suggests a question to which we do not know the answer: if $S$ is a scheme in positive characteristic satisfying some definition of rational singularities (such $F$-rationality), does Proposition 4.2 hold for $S$ ?

Example 4.6. We give an example to show that the regularity condition on $S$ cannot be weakened too much in Proposition 4.2. Let $(E, e) \subset \mathbb{P}^{2}$ be an elliptic curve, and let $S$ be the affine cone on $E$ with origin $s$. Note that $S$ is a hypersurface singularity of dimension 2 with 0 dimensional singular locus. In particular, it is normal. Let $A=S \times E$ denote the constant abelian scheme on $E$ over $S$. Then $U=S \backslash\{s\}$ can be identified with the total space of the $\mathbb{G}_{m}$-torsor $\left.\mathcal{O}(-1)\right|_{E}-0(E)$ over $E$. Thus, there exists a nonconstant section of $A(U)$. On the other hand, all sections $S \rightarrow A$ are constant. Indeed, every point in $S$ lies on an $\mathbb{A}^{1}$ containing $s$. As all maps $\mathbb{A}^{1} \rightarrow E$ are constant, the claim follows. Thus, we obtain an example of a normal hypersurface singularity $S$ and an abelian scheme $A \rightarrow S$ such that the conclusion of Proposition 4.2 fails for $S$. Of course, $S$ is not a rational singularity, a fact supported by Remark 4.5.

Next, we point out how to use Proposition 4.2 to prove the version of Theorem 1.2 involving Zariski cohomology under strong regularity assumptions on the base scheme $S$; the proof is trivial.

Corollary 4.7. Let $S$ be a regular excellent noetherian scheme, and let $f: A \rightarrow S$ be an abelian scheme. Then $H_{\mathrm{Zar}}^{n}(S, A)=0$ for $n>0$.

Proof. By Proposition 4.2, we know that $A$ restricts to a constant sheaf on the small Zariski site of each connected component of $S$. By the vanishing of the cohomology of a constant sheaf on an irreducible topological space, the claim follows.

We are now in a position to complete the proof of Theorem 1.2. 
Proof of Theorem 1.2. Let $S$ be a noetherian excellent scheme, and let $A \rightarrow S$ be an abelian scheme. We will show that cohomology classes in $H^{n}(S, A)$ are killed by proper surjective maps by induction on $n$ provided $n>0$. We may assume that $S$ is integral.

For $n=1$, classes in $H^{1}(S, A)$ are represented by étale $A$-torsors $T$ over $S$. As $T$ is an fppf $S$-scheme, there exists a quasifinite dominant morphism $U \rightarrow S$ such that $T(U)$ is nonempty. By picking an $S$-map $U \rightarrow T$ and taking the closure of the image, we obtain a proper surjective cover $S^{\prime} \rightarrow S$ such that $T\left(S^{\prime}\right)$ is not empty. This implies that the cohomology class associated to $T$ dies on passage to $S^{\prime}$, proving the claim.

We next proceed exactly as in the proof of Theorem 1.1 to reduce down to the case of a Čech cohomology class associated to a Zariski cover. The only difference is that the references to Proposition 3.1 should be replaced by references to Grothendieck's theorem [1968b, Théorème 11.7] which, in particular, implies that cohomology classes in $H_{\mathrm{fppf}}^{n}(S, A)$ trivialise over an étale cover; we omit the details.

To show the claim for a Čech cohomology class associated to a Zariski cover, assume first that $S$ is of finite type over $\mathbb{Z}$. In this case, thanks to de Jong's theorems [1997], we can find a proper surjective cover of $S$ with regular total space. Passing to this cover and applying Corollary 4.7 then solves the problem. In the case that $S$ is no longer of finite type over $\mathbb{Z}$, we reduce to the finite type case using approximation. Indeed, the data $(S, A, \alpha)$ comprising of the base scheme $S$, the abelian scheme $A \rightarrow S$, and a Čech cohomology class $\alpha \in H^{n}(U, A)$ associated to a finite Zariski open cover $U$ of $S$ can be approximated by similar data with all schemes involved of finite type over $\mathbb{Z}$. Given such an approximating triple $\left(S^{\prime}, A^{\prime}, \alpha^{\prime}\right)$ with $S^{\prime}$ of finite type over $\mathbb{Z}$, we can find a proper surjective map $S^{\prime \prime} \rightarrow S^{\prime}$ killing $\alpha^{\prime}$ by the earlier argument. By functoriality, the pullback $S^{\prime \prime} \times{ }_{S^{\prime}} S \rightarrow S$ is a proper surjective cover of $S$ killing $\alpha$.

Remark 4.8. Theorem 1.2 admits a topological reformulation as follows. Given a noetherian scheme $S$ and an abelian scheme $G$ over $S$, let $(\operatorname{Sch} / S)_{\text {prop }},(\operatorname{Sch} / S)_{\text {fppf }}$ and $(\mathrm{Sch} / S)_{\text {prop,fppf }}$ denote the (big) topoi associated to the category of schemes over $S$ equipped with the topology generated respectively by proper surjective maps, fppf maps, and both proper surjective and fppf maps. There are natural forgetful maps of topoi $a:(\mathrm{Sch} / S)_{\text {prop,fppf }} \rightarrow(\mathrm{Sch} / S)_{\text {prop }}$ and $b:(\mathrm{Sch} / S)_{\text {prop,fpp }} \rightarrow(\mathrm{Sch} / S)_{\text {fppf }}$ of topoi. Given an abelian scheme $G \rightarrow S$, let $G$ also denote the sheafification of the representable presheaf associated to $G$ in all of the above topologies. Theorem 1.2 can be reformulated as saying that the sheaves $\mathrm{R}^{i} a_{*} G$ vanish for $i>0$. Since schemes are sheaves for the fppf topology, one can easily show that $a_{*} a^{*} G=G$. Thus, Theorem 1.2 can be reformulated saying that $G \simeq \mathrm{R} a_{*} G$. Note a consequence: since cohomology on sites is computed using hypercovers by Verdier's theorem 
[Artin and Mazur 1969, Theorem 8.16], we see that for a class $\alpha \in H^{n}\left(S_{\mathrm{fppf}}, G\right)$ with $n>0$, there exists a proper hypercover $f_{\bullet}: T_{\bullet} \rightarrow S$ and a map of simplicial schemes $\phi: T_{\bullet} \rightarrow K(G, n)$ representing $b^{*} \alpha$. If $G$ is instead a finite flat group scheme, then the same remarks apply for Theorem 1.1, except that we replace proper maps by finite ones.

\section{An application: big Cohen-Macaulay algebras in positive characteristic}

Let $(R, \mathfrak{m})$ be an excellent noetherian local domain containing $\mathbb{F}_{p}$. A fundamental theorem of Hochster-Huneke [1992] asserts that the absolute integral closure $R^{+}$ (the integral closure of $R$ in a fixed algebraic closure of its fraction field) is a Cohen-Macaulay algebra. This result and the ideas informing it form the bedrock of tight closure theory and huge swathes of positive characteristic commutative algebra.

Our goal in this section is to give a new proof of the Hochster-Huneke theorem using Theorem 1.1. We hasten to remark that there already exist alternative proofs in the literature, all cocycle-theoretic or equational at the core. The approach adopted here follows closely the relatively recent approach from [Huneke and Lyubeznik 2007], the essential new feature being the use of cohomology-annihilation result proven in Theorem 1.1 in place of explicit cocycle manipulations.

We begin by recording a coherent cohomology-annihilation result one can deduce from Theorem 1.1; this can be considered as the analogue of the "equational lemma" of [Hochster and Huneke 1992]; see also [Huneke and Lyubeznik 2007, Lemma 2.2].

Proposition 5.1. Let $(R, \mathfrak{m})$ be a noetherian excellent local $\mathbb{F}_{p}$-algebra, and let $M \subset H_{\mathfrak{m}}^{i}(R)$ be a Frobenius stable finite length $R$-submodule for some $i>0$. Then there exists a module-finite extension $f: R \rightarrow S$ such that $f^{*}(M)=0$ where $f^{*}: H_{\mathfrak{m}}^{i}(R) \rightarrow H_{\mathfrak{m}}^{i}(S)$ is the induced map.

Proof. After normalising $R$, we may assume that $i>1$. With $U=\operatorname{Spec}(R)-\{\mathfrak{m}\}$, we have a Frobenius equivariant identification

$$
H^{i-1}(U, \mathcal{O}) \simeq H_{\mathfrak{m}}^{i}(R)
$$

which allows us to view $M$ as a submodule of $H^{i-1}(U, \mathcal{O})$. The Frobenius action endows $H^{i-1}(U, \mathcal{O})$ with the structure of a $R\left\{X^{p}\right\}$-module, where $R\left\{X^{p}\right\}$ is the noncommutative polynomial ring over $R$ with one generator $X^{p}$ satisfying the commutation relation $X^{p} r=r^{p} X^{p}$ for $r \in R$. The finite length assumption implies that for each $m \in M$, there exists some monic additive polynomial $g\left(X^{p}\right) \in R\left\{X^{p}\right\}$ such that $g(m)=0$. As $g$ is additive and monic, we have a short exact sequence

$$
0 \rightarrow \operatorname{ker}(g) \rightarrow \mathrm{O} \stackrel{g}{\rightarrow} \mathrm{O} \rightarrow 0
$$


of abelian sheaves on $\operatorname{Spec}(R)_{\mathrm{fppf}}$. Moreover, the monicity of $g$ also shows that the sheaf $\operatorname{ker}(g)$ is representable by a finite flat commutative group scheme over $\operatorname{Spec}(R)$. As $g(m)=0$, we see that $m$ comes from a cohomology class $m^{\prime} \in$ $H^{i-1}(U, \operatorname{ker}(g))$. Since $i-1>0$, Theorem 1.1 shows that there exists a finite surjective map $\pi: V \rightarrow U$ such that $\pi^{*} m^{\prime}=0$. Setting $S$ to be the (global sections of the) normalisation of $R$ in $V$ is then seen to solve the problem.

Using Proposition 5.1, we can give a proof that $R^{+}$is Cohen-Macaulay. The argument given below is based entirely on [Huneke and Lyubeznik 2007, Theorem 2.1] and simply recorded here for convenience.

Theorem 5.2. Let $(R, \mathfrak{m})$ be a noetherian excellent local $\mathbb{F}_{p}$-domain that admits a dualising complex, and let $R^{+}$be its absolute integral closure. Then $R^{+}$is Cohen-Macaulay.

Proof. We first briefly review local duality and set up some notation. The local duality functor $D$ (sometimes referred to as Matlis duality) is defined by $\operatorname{Hom}_{R}(-, E)$ where $E$ is an injective hull of the residue field $R / \mathfrak{m}$. Once a dualising complex $\omega_{R}^{\bullet}$ has been fixed (normalised as usual to have the dualising sheaf $\omega_{R}$ in homological degree $\operatorname{dim}(R))$, the hull $E$ may be identified with $\mathrm{R} \Gamma_{\mathfrak{m}}\left(\omega_{R}^{\bullet}\right)$ in $D^{b}(R)$. This functor is exact, contravariant, length preserving (on finite length $R$-modules), and transforms ind-artinian $R$-modules to pro-artinian $R$-modules. Moreover, local duality asserts that for finite $R$-modules $M$, one has $D\left(\operatorname{Ext}_{R}^{-i}\left(M, \omega_{R}^{\bullet}\right)\right) \simeq H_{\mathfrak{m}}^{i}(M)$ and

$$
\left.D\left(H_{\mathfrak{m}}^{i}(M)\right) \simeq \operatorname{Ext}_{R} \widehat{-i(M,} \omega_{R}^{\bullet}\right)
$$

where $\widehat{N}$ denotes the m-adic completion of $N$. For more details, see [Hartshorne 1966, Chapter V, §6; Brodmann and Sharp 1998, §10].

We need the compatibility between duality and localisation, which we recall next. Let $d=\operatorname{dim}(R)$, and for a prime $\mathfrak{p} \in \operatorname{Spec}(R)$, we set $d_{\mathfrak{p}}=\operatorname{dim}\left(R_{\mathfrak{p}}\right)$ and $c_{\mathfrak{p}}=d-d_{\mathfrak{p}}$. One has $\left(\omega_{R}^{\bullet}\right)_{\mathfrak{p}} \simeq \omega_{R_{\mathfrak{p}}}^{\bullet}\left[c_{\mathfrak{p}}\right]$, which leads to the formula

$$
\operatorname{Ext}_{R}^{-i}\left(M, \omega_{R}^{\bullet}\right)_{\mathfrak{p}} \simeq \operatorname{Ext}_{R_{\mathfrak{p}}}^{c_{\mathfrak{p}}-i}\left(M_{\mathfrak{p}}, \omega_{R_{\mathfrak{p}}}^{\bullet}\right)
$$

for finite $R$-modules $M$. This gives a direct connection between $H_{\mathfrak{m}}^{i}(R)$ and $H_{\mathfrak{p}}^{i-c_{\mathfrak{p}}}\left(R_{\mathfrak{p}}\right)$ which will be exploited in the proof below; see [Grothendieck 1968a, Exposé VIII, Théorème 2.1] for another application.

To show that $R^{+}$is Cohen-Macaulay, we will show by induction on $d$ that there exists a module-finite extension $R \rightarrow S$ which kills local cohomology outside degree $d$. By the local duality as explained above, it suffices to find a module-finite extension $R \rightarrow S$ such that the induced map $\operatorname{Ext}_{R}^{-i}\left(S, \omega_{R}^{\bullet}\right) \rightarrow \operatorname{Ext}_{R}^{-i}\left(R, \omega_{R}^{\bullet}\right)$ is 0 for $i<d$. The case $d=0$ being vacuous, we assume $d>0$ and pick a nonnegative integer $i<d$. Let $\mathfrak{p}_{1}, \ldots, \mathfrak{p}_{n}$ be the set of all of nonmaximal associated primes 
of $\operatorname{Ext}_{R}^{-i}\left(R, \omega_{R}^{\bullet}\right)$. For each such prime $\mathfrak{p}_{j}$, induction constructs a module-finite extension $R_{\mathfrak{p}_{j}} \rightarrow S_{j}$ that kills $H_{\mathfrak{p}_{j}}^{i-c_{\mathfrak{p}_{j}}}\left(R_{\mathfrak{p}_{j}}\right)$; note that $i-c_{\mathfrak{p}_{j}}<d_{\mathfrak{p}_{j}}$ since $i<d$. Setting $S$ to be the normalisation of $R$ in a compositum of all the $S_{j}$ then shows that the map $R \rightarrow S$ induces a map $f_{*}: \operatorname{Ext}_{R}^{-i}\left(S, \omega_{R}^{\bullet}\right) \rightarrow \operatorname{Ext}_{R}^{-i}\left(R, \omega_{R}^{\bullet}\right)$ whose localisation at $\mathfrak{p}_{j}$ is the 0 map (as it is a map of finite $R_{\mathfrak{p}_{j}}$-modules that is 0 after $\mathfrak{p}_{j}$ adic completion). Since the only other possible associated prime of $\operatorname{Ext}_{R}^{-i}\left(R, \omega_{R}^{\bullet}\right)$ is $\mathfrak{m}$, it follows that $\operatorname{im}\left(f_{*}\right)$ is a finite length submodule of $\operatorname{Ext}_{R}^{-i}\left(R, \omega_{R}^{\bullet}\right)$. By duality, the image of $f^{*}: H_{\mathfrak{m}}^{i}(R) \rightarrow H_{\mathfrak{m}}^{i}(S)$ is a finite length $R$-submodule $M$ of $H_{\mathfrak{m}}^{i}(S)$ which is moreover Frobenius stable. Hence, the $S$-submodule of $H_{\mathfrak{m}}^{i}(S) \simeq H_{\mathfrak{m} S}^{i}(S)$ generated by $M$ is also Frobenius stable with finite length. Since $\mathfrak{m} S$ is a finite colength ideal in $S$, Proposition 5.1 gives a module-finite extension $S \rightarrow T$ killing $M$; the composite $R \rightarrow T$ then kills $H_{\mathfrak{m}}^{i}(R)$.

\section{An example of a torsor not killed by finite covers}

Theorem 1.2 lets one construct proper covers annihilating cohomology with coefficients in an abelian scheme. We will show that "proper" cannot be replaced by "finite" in the preceding statement.

Example 6.1. In [Raynaud 1970, Example 3.2, Chapter XIII], one finds an example of a semilocal normal connected noetherian affine $S$ of dimension 2, an abelian scheme $A \rightarrow S$, and an $A$-torsor $X \rightarrow S$ (in the category of fppf sheaves) that is Zariski locally trivial, and defines an infinite order element $\alpha \in H^{1}(S, A)$. By transfers (see Corollary 6.3), it follows that $\alpha$ cannot be trivialised by passing to a finite cover $T \rightarrow S$.

Example 6.1 relies on the existence of certain "transfer" maps whose construction we now explain. Fix a normal connected base scheme $S$, and a locally finitely presented algebraic space $A \rightarrow S$ that represents an abelian sheaf on the category of $S$-schemes. Our goal is to explain why étale cohomology with coefficients in $A$ carries natural pushforward maps. As a corollary, when $A \rightarrow S$ is smooth, we obtain pushforwards maps in fppf cohomology as well.

Proposition 6.2. Let $f: T \rightarrow S$ be a finite surjective map with $T$ normal and equidimensional. There exists a map of abelian sheaves $\operatorname{Nm}(f): f_{*} f^{*} A \rightarrow A$ on the small étale site of $S$ such that the composite

$$
A \stackrel{f^{*}}{\longrightarrow} f_{*} f^{*} A \stackrel{\mathrm{Nm}(f)}{\longrightarrow} A
$$

is multiplication by $d=\operatorname{deg}(f)$.

Proposition 6.2 is well-known, but we do not know a reference, so we sketch a proof. 
Sketch of proof. We first construct the map on global sections, and then show it sheafifies.

Assume first that $T$ is connected, and $f: T \rightarrow S$ is generically Galois with group $G$ of cardinality $d$. By normality of $S$, we identify $T / G \simeq S$, where the quotient $T / G$ is computed in the category of algebraic spaces (or schemes). Given a $T$-point $a \in A(T)$, we obtain a natural map $T \rightarrow \operatorname{Map}(G, A) \simeq A^{d}$ given by $t \mapsto(g \mapsto a(g(t)))$. The group $S_{d}=S_{\# G}$ acts on $\operatorname{Map}(G, A)$, and the preceding map $T \rightarrow \operatorname{Map}(G, A)$ is equivariant for the natural embedding $G \rightarrow S_{d}$ given by left translation. Taking quotients as algebraic spaces, we get a map

$$
b: S \simeq T / G \rightarrow A^{d} / S_{d}=\operatorname{Sym}^{d}(A) .
$$

The $d$-fold multiplication map $A^{d} \rightarrow A$ is an $S_{d}$-equivariant map to an algebraic space, and hence factors as $A^{d} \rightarrow \operatorname{Sym}^{d}(A) \stackrel{m}{\longrightarrow} A$. Composing $m$ with $b$ gives a map $S \rightarrow A$ that we declare to be the norm $\operatorname{Nm}(f)(S)(a)$.

Assume now that $T$ is connected and $f: T \rightarrow S$ has degree $d$, but is not necessarily generically Galois. Then the arguments of [Suslin and Voevodsky 1996, Theorem 6.7, page 81] ensure that $\operatorname{Sym}^{d}(T) \rightarrow S$ has a natural section $s$ constructed from $f$. Given $a \in A(T)$, we obtain a map

$$
S \stackrel{s}{\rightarrow} \operatorname{Sym}^{d}(T) \stackrel{\operatorname{Sym}^{d}(a)}{\longrightarrow} \operatorname{Sym}^{d}(A) \stackrel{m}{\rightarrow} A
$$

that we declare to be the norm $\operatorname{Nm}(f)(S)(a)$.

In the general case, we perform the preceding construction on each connected component of $T$. The composite map $A(S) \rightarrow A(T) \rightarrow A(S)$ is easily seen to be multiplication by $d$ using the fact that $A \stackrel{\Delta}{\longrightarrow} A^{d} \rightarrow \operatorname{Sym}^{d}(A) \stackrel{m}{\longrightarrow} A$ is multiplication by $d$. Finally, we observe that all hypotheses are stable under étale base change on $S$, so the preceding construction gives map of sheaves on the small étale site of $S$.

Corollary 6.3. Let $f: T \rightarrow S$ be a finite surjective morphism with $T$ normal and equidimensional. Assume that $A \rightarrow S$ is smooth. Then there exist pushforward maps $H_{\mathrm{fppf}}^{i}(T, A) \rightarrow H_{\mathrm{fppf}}^{i}(S, A)$ such that the composite

$$
H_{\mathrm{fppf}}^{i}(S, A) \rightarrow H_{\mathrm{fppf}}^{i}(T, A) \rightarrow H_{\mathrm{fppf}}^{i}(S, A)
$$

is multiplication by $d$.

Proof. Let $f^{\text {ét }}: T_{\text {ét }} \rightarrow S_{\text {ét }}$ be the induced map of small étale sites. Acyclicity for finite morphisms shows that $H_{\text {êt }}^{i}\left(S, f_{*}^{\text {ét }} f^{\text {ét,*}} A\right)=H_{\text {êt }}^{i}\left(T, f^{\text {ét,*}} A\right)$. Grothendieck's theorem [1968b, Théorème 11.7] and the smoothness of $A$ show that

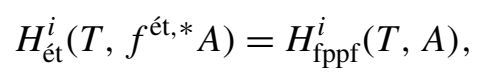

where the right hand side is defined by viewing $A$ as a sheaf on the big fppf site of $(\mathrm{Sch} / S)_{\text {fppf. }}$ The claim now follows from Proposition 6.2. 


\section{Acknowledgements}

The results presented here formed a part of my doctoral thesis written under Johan de Jong, and I would like to thank him for stimulating discussions. I would also like to thank János Kollár for a very useful conversation, and Brian Conrad for suggesting simplifications to some convoluted arguments (especially in Proposition 3.1) in an earlier version of the note. Finally, thanks are due to the anonymous referee for spotting gaps and errors in an earlier version of this preprint.

\section{References}

[Artin and Mazur 1969] M. Artin and B. Mazur, Étale homotopy, Lecture Notes in Mathematics 100, Springer, Berlin, 1969. MR 39 \#6883 Zbl 0182.26001

[Berthelot et al. 1982] P. Berthelot, L. Breen, and W. Messing, Théorie de Dieudonné cristalline, II, Lecture Notes in Mathematics 930, Springer, Berlin, 1982. MR 85k:14023 Zbl 0516.14015

[Brodmann and Sharp 1998] M. P. Brodmann and R. Y. Sharp, Local cohomology: an algebraic introduction with geometric applications, Cambridge Studies in Advanced Mathematics 60, Cambridge University Press, 1998. MR 99h:13020 Zbl 0903.13006

[Conrad 2007] B. Conrad, "Deligne's notes on Nagata compactifications", J. Ramanujan Math. Soc. 22:3 (2007), 205-257. MR 2009d:14002 Zbl 1142.14001

[Deligne 1977] P. Deligne, Cohomologie étale (SGA 4 $\frac{1}{2}$ ), Lecture Notes in Math. 569, Springer, Berlin, 1977. MR 57 \#3132

[Fontaine 1994] J.-M. Fontaine, "Le corps des périodes p-adiques", pp. 59-111 Astérisque 223, Société Mathématique de France, Paris, 1994. MR 95k:11086 Zbl 0940.14012

[Grothendieck 1962] A. Grothendieck, Revêtements étales et groupe fondamental: Séminaire de géométrie algébrique du Bois Marie 1960-61 (SGA 1), Lecture Notes in Math. 224, Springer, Berlin, 1962. Reprinted Soc. math. de France, Paris, 2003. MR 50 \#7129 Zbl 1039.14001

[Grothendieck 1966] A. Grothendieck, "Éléments de géométrie algébrique, IV: Étude locale des schémas et des morphismes de schémas, III", Inst. Hautes Études Sci. Publ. Math. 28 (1966), 255. MR 36 \#178 Zbl 0144.19904

[Grothendieck 1968a] A. Grothendieck, Cohomologie locale des faisceaux cohérents et théorèmes de Lefschetz locaux et globaux (SGA 2), Advanced Stud. in Pure Math., North-Holland, Amsterdam, 1968. MR 57 \#16294 Zbl 0159.50402

[Grothendieck 1968b] A. Grothendieck, "Le groupe de Brauer, III: exemples et compléments", pp. 88-188 in Dix exposés sur la cohomologie des schémas, North-Holland, Amsterdam, 1968. MR 39 \#5586c Zbl 0198.25901

[Hartshorne 1966] R. Hartshorne, Residues and duality, Lecture Notes in Mathematics 20, Springer, Berlin, 1966. MR 36 \#5145 Zbl 0212.26101

[Hochster 2007] M. Hochster, "Homological conjectures, old and new", Illinois J. Math. 51:1 (2007), 151-169. MR 2008j:13034 Zbl 1127.13010

[Hochster and Huneke 1992] M. Hochster and C. Huneke, "Infinite integral extensions and big CohenMacaulay algebras", Ann. of Math. (2) 135:1 (1992), 53-89. MR 92m:13023 Zbl 0753.13003

[Hoobler 1982] R. T. Hoobler, "When is $\operatorname{Br}(X)=\mathrm{Br}^{\prime}(X)$ ?", pp. 231-244 in Brauer groups in ring theory and algebraic geometry (Wilrijk, 1981), edited by F. M. J. van Oystaeyen and A. H. Verschoren, Lecture Notes in Math. 917, Springer, Berlin, 1982. MR 83g:14006 Zbl 0491.14013 
[Huneke and Lyubeznik 2007] C. Huneke and G. Lyubeznik, "Absolute integral closure in positive characteristic”, Adv. Math. 210:2 (2007), 498-504. MR 2008d:13005 Zbl 1109.13003

[de Jong 1997] A. J. de Jong, "Families of curves and alterations", Ann. Inst. Fourier (Grenoble) 47:2 (1997), 599-621. MR 98f:14019 Zbl 0868.14012

[Kollár 1996] J. Kollár, Rational curves on algebraic varieties, Ergebnisse der Math. (3) 32, Springer, Berlin, 1996. MR 98c:14001 Zbl 0877.14012

[Messing 1972] W. Messing, The crystals associated to Barsotti-Tate groups: with applications to abelian schemes, Lecture Notes in Mathematics 264, Springer, Berlin, 1972. MR 50 \#337 Zbl 0243.14013

[Milne 2008] J. Milne, “Abelian varieties”, preprint, 2008, http://www.jmilne.org/math/CourseNotes/ av.html.

[Mumford et al. 1994] D. Mumford, J. Fogarty, and F. Kirwan, Geometric invariant theory, 3rd ed., Ergebnisse der Math. (2) 34, Springer, Berlin, 1994. MR 95m:14012 Zbl 0797.14004

[Raynaud 1967] M. Raynaud, "Sur le passage au quotient par un groupoïde plat", C. R. Acad. Sci. Paris Sér. A-B 265 (1967), A384-A387. MR 36 \#5147 Zbl 0193.48901

[Raynaud 1970] M. Raynaud, Faisceaux amples sur les schémas en groupes et les espaces homogènes, Lecture Notes in Mathematics 119, Springer, Berlin, 1970. MR 41 \#5381 Zbl 0195.22701

[Stacks] Stacks, The Stacks project, http://math.columbia.edu/algebraic_geometry/stacks-git/.

[Suslin and Voevodsky 1996] A. Suslin and V. Voevodsky, "Singular homology of abstract algebraic varieties", Invent. Math. 123:1 (1996), 61-94. MR 97e:14030 Zbl 0896.55002

[Tate and Oort 1970] J. Tate and F. Oort, "Group schemes of prime order", Ann. Sci. École Norm. Sup. (4) 3 (1970), 1-21. MR 42 \#278 Zbl 0195.50801

Communicated by Brian Conrad

Received 2011-09-26 Revised 2011-12-06 Accepted 2012-01-03

bhattb@umich.edu

Department of Mathematics, University of Michigan, 2074 East Hall, 530 Church Street, Ann Arbor, MI 48109, United States

Current address: $\quad$ School of Mathematics, Institute for Advanced Study, Princeton, NJ 08540, United States http://www-personal.umich.edu/ bhattb/ 


\section{Algebra \& Number Theory}

msp.berkeley.edu/ant

\section{EDITORS}

MANAGING EDITOR

Bjorn Poonen

Massachusetts Institute of Technology

Cambridge, USA

\author{
EDITORIAL BOARD CHAIR \\ David Eisenbud \\ University of California \\ Berkeley, USA
}

\section{BOARD OF EDITORS}

Georgia Benkart

Dave Benson

Richard E. Borcherds

John H. Coates

J-L. Colliot-Thélène

Brian D. Conrad

Hélène Esnault

Hubert Flenner

Edward Frenkel

Andrew Granville

Joseph Gubeladze

Ehud Hrushovski

Craig Huneke

Mikhail Kapranov

Yujiro Kawamata

János Kollár

Yuri Manin

Barry Mazur

Philippe Michel
University of Wisconsin, Madison, USA

University of Aberdeen, Scotland

University of California, Berkeley, USA

University of Cambridge, UK

CNRS, Université Paris-Sud, France

University of Michigan, USA

Freie Universität Berlin, Germany

Ruhr-Universität, Germany

University of California, Berkeley, USA

Université de Montréal, Canada

San Francisco State University, USA

Hebrew University, Israel

University of Virginia, USA

Yale University, USA

University of Tokyo, Japan

Princeton University, USA

Northwestern University, USA

Harvard University, USA

École Polytechnique Fédérale de Lausanne
Susan Montgomery

Shigefumi Mori

Raman Parimala

Jonathan Pila

Victor Reiner

Karl Rubin

Peter Sarnak

Joseph H. Silverman

Michael Singer

Vasudevan Srinivas

J. Toby Stafford

Bernd Sturmfels

Richard Taylor

Ravi Vakil

Michel van den Bergh

Marie-France Vignéras

Kei-Ichi Watanabe

Andrei Zelevinsky

Efim Zelmanov
University of Southern California, USA

RIMS, Kyoto University, Japan

Emory University, USA

University of Oxford, UK

University of Minnesota, USA

University of California, Irvine, USA

Princeton University, USA

Brown University, USA

North Carolina State University, USA

Tata Inst. of Fund. Research, India

University of Michigan, USA

University of California, Berkeley, USA

Harvard University, USA

Stanford University, USA

Hasselt University, Belgium

Université Paris VII, France

Nihon University, Japan

Northeastern University, USA

University of California, San Diego, USA

\section{PRODUCTION}

production@msp.org

Silvio Levy, Scientific Editor

See inside back cover or www.jant.org for submission instructions.

The subscription price for 2012 is US \$175/year for the electronic version, and \$275/year ( $\$ 40$ shipping outside the US) for print and electronic. Subscriptions, requests for back issues from the last three years and changes of subscribers address should be sent to Mathematical Sciences Publishers, Department of Mathematics, University of California, Berkeley, CA 94720-3840, USA.

Algebra \& Number Theory (ISSN 1937-0652) at Mathematical Sciences Publishers, Department of Mathematics, University of California, Berkeley, CA 94720-3840 is published continuously online. Periodical rate postage paid at Berkeley, CA 94704, and additional mailing offices.

ANT peer review and production are managed by EditFLOW ${ }^{\circledR}$ from Mathematical Sciences Publishers.

PUBLISHED BY

mathematical sciences publishers

http://msp.org/

A NON-PROFIT CORPORATION

Typeset in IATEX

Copyright ( 2012 by Mathematical Sciences Publishers 


\section{Algebra \& Number Theory}

\section{Volume $6 \quad$ No. $7 \quad 2012$}

On the rank of the fibers of rational elliptic surfaces

Cecília Salgado

Néron's pairing and relative algebraic equivalence

CÉDRIC PÉPIN

Free subalgebras of quotient rings of Ore extensions

JASON P. BELL and DANIEL ROGALSKI

Classes de cycles motiviques étales

1369

BRUNO KAHN

Higher-order Maass forms

1409

ROELOF BRUGGEMAN and NiKOLAOS DIAMANTIS

Log canonical thresholds, $F$-pure thresholds, and nonstandard extensions

Bhargav Bhatt, Daniel J. Hernández, Lance Edward Miller and Mircea MUSTAȚĂ

The semistable reduction problem for the space of morphisms on $\mathbb{P}^{n}$

ALON LEVY

Grothendieck's trace map for arithmetic surfaces via residues and higher adèles

MATTHEW MORROW

Crystalline extensions and the weight part of Serre's conjecture

Toby GeE, Tong LiU and DAVID SAVITT

Annihilating the cohomology of group schemes 